\title{
Vertical composition gradient in InGaAs/GaAs alloy quantum dots as revealed by high-resolution x-ray diffraction
}

\author{
M. Hanke ${ }^{a)}$ \\ Martin-Luther-Universität Halle-Wittenberg, Hoher Weg 8, D-06120 Halle(Saale), Germany \\ D. Grigoriev, M. Schmidbauer, P. Schäfer, and R. Köhler \\ Institut für Physik, Humboldt-Universität zu Berlin, Newtonstraße 15, D-12489 Berlin, Germany \\ R. L. Sellin, ${ }^{\text {b) }}$ U. W. Pohl, and D. Bimberg \\ Institut für Festkörperphysik, Technische Universität Berlin, Hardenbergstraße 36, D-10623 Berlin, \\ Germany
}

(Received 6 July 2004; accepted 9 August 2004)

\begin{abstract}
Shape and composition profiles of self-organized $\mathrm{In}_{0.6} \mathrm{Ga}_{0.4} \mathrm{As} / \mathrm{GaAs}$ quantum dots (QDs) were investigated using diffuse x-ray scattering of a fivefold QD stack. To reveal the QD morphology, numerical scattering simulations of QDs with different morphologies were performed based on three-dimensional strain fields calculated by the finite element methods. Comparing our simulations to the data, we proved that the In concentration increases from the wetting layer to the top of the quantum dots. Moreover, we conclude that the In concentration of the wetting layers is significantly lower than the average value in the QDs. () 2004 American Institute of Physics.
\end{abstract}

[DOI: $10.1063 / 1.1803938]$

Self-organized InGaAs/GaAs quantum dots (QDs) as formed in the Stranski-Krastanow growth mode are zerodimensional electronic systems, ${ }^{1,2}$ which are highly interesting for both the study of new physical phenomena and device applications, such as lasers and semiconductor optical amplifiers (SOAs). ${ }^{3}$ Recent studies on the electronic and optical properties of QDs have outlined the impact of QD shape, chemical composition and strain profile on the alignment of confined electron and hole wave functions, ${ }^{4}$ as well as the dependence of the polarization of emitted photons on the QD aspect ratio. ${ }^{5}$ While the morphological properties of QDs were thus shown to be decisive for the design of QD devices, such as polarization-insensitive SOAs, the debate on the In distribution within InGaAs alloy QDs, as well as the exact shape of such QDs, is still ongoing.

Various techniques have been applied to reveal both QD strain and composition profiles. High-resolution transmission electron microscopy (HRTEM) is mostly applied to monitor chemical and strain contrast. The technique allows for atomic resolution, but a clear separation of strain and chemical composition contrast is difficult. Moreover, only columns of atoms are imaged, which average compositional variations along the direction of projection. Cross-sectional tunneling microscopy (XSTM) enables to image strain contrast and compositional profiles with atomic resolution on cleaved surfaces across buried QDs. Using XSTM, different morphologies of InGaAs/GaAs QDs have been identified, including reverse-trapezoidal ${ }^{6}$ and reverse-pyramidal ${ }^{7,8}$ In-rich cores.

Direct imaging techniques, such as HRTEM or XSTM, require sample preparation which alters the shape and strain profile of the investigated QDs. Furthermore, these techniques provide data on only a few selected objects which do

\footnotetext{
${ }^{\text {a) Present address: National Institute of Standards and Technology, } 100 \mathrm{Bu}-}$ reau Drive, Gaithersburg, MD 20899, USA; electronic address: hanke@physik.uni-halle.de

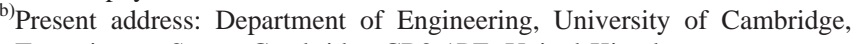
Trumpington Street, Cambridge CB2 1PZ, United Kingdom.
}

not necessarily represent the QD ensemble. In this letter we show that diffuse x-ray scattering, accompanied by numerical scattering simulations, ${ }^{9,10}$ can likewise be used to reveal inhomogeneous composition profiles in InGaAs/GaAs QDs. The technique is nondestructive and leaves QDs in their original strain environment. Moreover, data are collected on entire QD ensembles, thereby avoiding characterization of potentially unrepresentative QDs.

The sample investigated here was grown on a GaAs(001) substrate by metalorganic chemical-vapor deposition using trimethylgallium, trimethylindium, and arsine as precursors. Each QD layer was grown at $500{ }^{\circ} \mathrm{C}$, whereas the temperature was increased during GaAs overgrowth to $600{ }^{\circ} \mathrm{C}$. In order to stack subsequent layers closely with a vertical period around $20 \mathrm{~nm}$, we applied a surface-flattening technique during growth of the respective GaAs spacer layers. ${ }^{11}$

Transmission electron micrographs (not shown here) depict that the QDs reside on a very thin and continuous wetting layer (WL). Enforced by the long-range strain field of the QDs and the comparatively thin GaAs spacers between the dot planes, the QDs are vertically arranged into columns, whereas they do not exhibit a significant lateral ordering. ${ }^{12}$ Highly brilliant $\mathrm{x}$-ray radiation is mandatory to yield sufficient scattered intensity from the QDs, owing to their small volume. We therefore used monochromatic synchrotron radiation from the beamline BW2 at HASYLAB with an energy of $8 \mathrm{keV}$ and an energy resolution of $\Delta E / E=10^{-4}$. A position-sensitive detector which simultaneously records the intensity along a curved line in reciprocal space was used for recording two-dimensional intensity distributions. Our procedure for the numerical simulations is based on an iterative two-step approach. First, the three-dimensional strain field is calculated applying the finite element method (FEM), which basically employs linear elasticity theory. Thus, the full elastic anisotropy of the zincblende structure is considered. Eventually, the derived deformation fields serve as data 


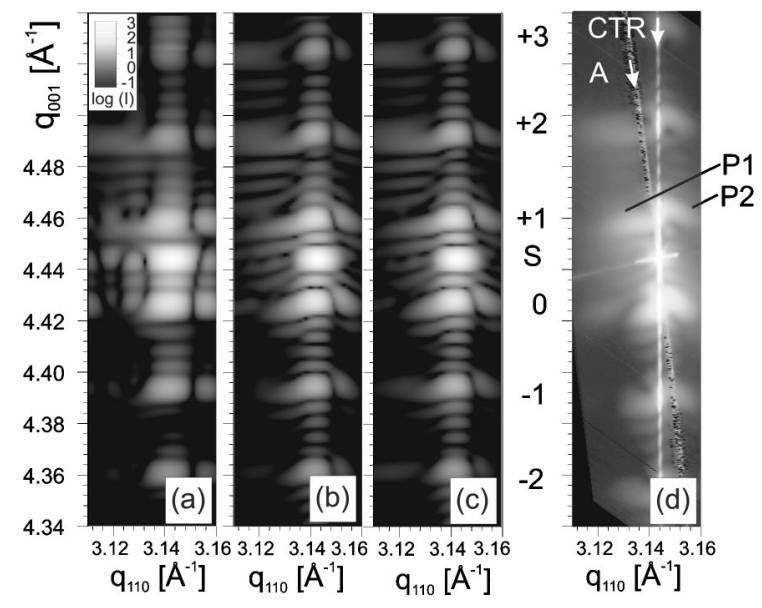

FIG. 1. Measured x-ray intensity distribution in the vicinity of the asymmetrical 224 reciprocal lattice point (d) compared to simulations of the scattered intensity calculated using different QD shapes and composition profiles $(\mathrm{a}-\mathrm{c})$ as described in Fig. 3. Feature (A) is caused by a detector artefact.

input for numerical scattering simulations on the basis of the distorted-wave Born approximation. ${ }^{9}$

Figure 1 shows a reciprocal space map around the GaAs 224 reflection (d) and simulated intensity distributions (a-c) for different QD shapes and compositions as described later. The diffuse x-ray scattering in Fig. 1(d) exhibits a complicated pattern which results from the simultaneous action of geometry, strain, and chemical composition profile within the QDs. Moreover, the signal contains contributions from the InGaAs WLs and the GaAs matrix. The GaAs substrate reflection $(S)$ appears as a very strong signal at $q_{001}$ $=4.445 \AA^{-1}$. It is vertically intersected at $q_{110}=3.143 \AA^{-1}$ by the crystal truncation rod (CTR), which appears as a bright line. The equally spaced numbered satellites on the CTR originate from the vertical periodicity of the sample structure. Their vertical spacing of $0.0327 \AA^{-1}$ reveals a QD layer-to-layer period of $19.2 \mathrm{~nm}$, in good agreement with the nominal value of $20 \mathrm{~nm}$. The weaker ancillary maxima between these satellites reflect the overall thickness of the fivefold QD stack.

The laterally extended and homogeneous sample regions of the GaAs matrix and the WLs between the QD columns solely contribute to the intensity on and close to the CTR, because the lateral lattice parameter here is very close to that of the GaAs substrate, and thus only vertical strain exists in that area. This is evidenced by a simulation of the lateral strain tensor component $\epsilon_{x x}$ across one QD column as shown in Fig. 2(a) and the vertical strain tensor component $\epsilon_{z z}$ as shown in Fig. 2(b). Since the volume of the QDs is small as compared to that of the remaining sample structure, the intensity distribution on and close to the CTR is strongly dominated by scattering from the extended regions between the QD columns. Consequently, the vertical position of the zeroorder satellite on the CTR at $q_{001}=4.427 \AA^{-1}$ enables a measure of the mean In content of the WLs: it is assessed to be 2 GaAs lattice constants $a=5.6532-\AA$ thick and to contain $37 \%$ In. It must be noted that variations of the WL thickness in the simulations essentially alter the intensities of higher-order satellites only, provided that the product of WL thickness and In concentration is kept constant. The assumed thickness corresponds to values previously reported for similar InGaAs/GaAs QD stacks ${ }^{12}$ as revealed by transmission elec-
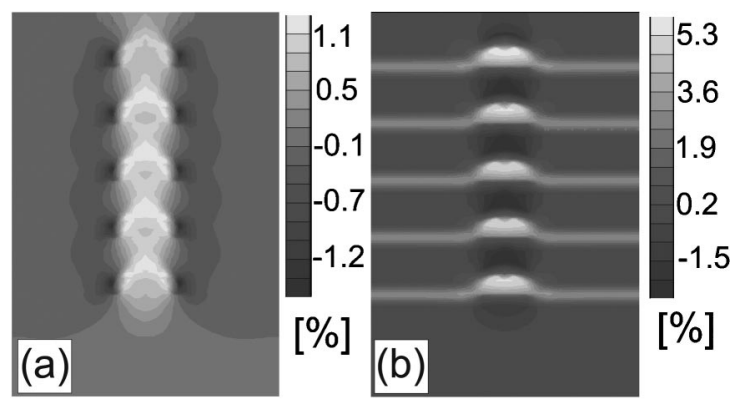

FIG. 2. Maps of the total strain tensor components $\epsilon_{x x}$ (a) and $\epsilon_{z z}$ (b) for a QD as shown in Fig. 3(b), calculated using the finite element method. Relative strain values as denoted in the scale refer to the GaAs lattice parameters.

tron microscopy. The In concentration of $37 \%$ is considerably smaller than the nominal value of $60 \%$. This difference can be explained by In migration from the WL towards the QDs during island formation ${ }^{13,14}$ as substantiated later.

Figure 2(a) shows that only the dot stack comprises regions of nonzero lateral strain. Therefore, only these regions give rise to the diffuse scattering on both sides of the CTR. This makes the diffuse signal an appropriate means to probe the In distribution within the QDs and the transition regions to the adjacent GaAs matrix. The diffuse scattering shows a rather complicated intensity pattern; note, e.g., shape and orientation of the features $P 1$ and $P 2$ in Fig. 1(d), which show the effect of locally compressed and dilatated regions, respectively. The complexity of the intensity distribution prevents straightforward conclusions on the QD morphology. To extract quantitative information, we performed dynamical scattering simulations probing different QD shapes, namely cuboids, ${ }^{12}$ pyramids, flat lenses, and inverted cones. In turn, different In concentration profiles were assumed for the respective shapes. Figure 3 shows cross-sectional schemes of three selected model QDs we simulated in this work. A lenslike QD with 60\% In content on top of a WL is shown in Fig. 3(a). The second model QD shown in Fig. 3(b) has the same shape, size, and In concentration as the previous one, but

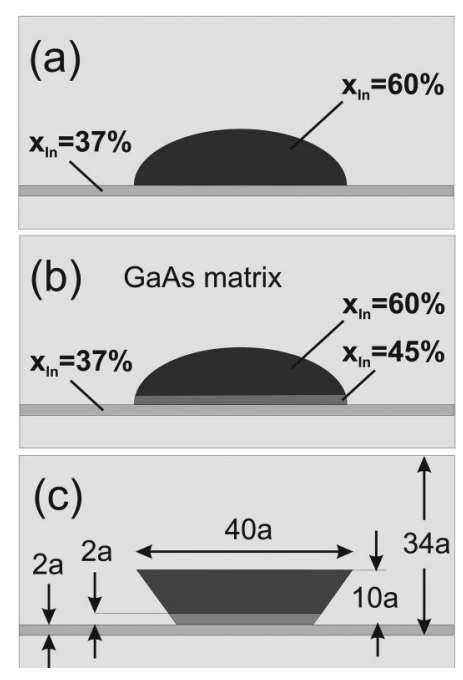

FIG. 3. Model QD shapes and compositions used for scattering simulations. The wetting layer contains $37 \%$ In in all three structures. (a) Lens-shaped QD with homogeneous QD apex, containing 60\% In. (b) Lens-like QD with a $2 \times a$ GaAs thick transition layer containing $45 \%$ In. (c) Inverted-coneshaped QD with a $2 \times a$ GaAs transition layer containing $45 \%$ In. Dot dimensions are given in units of the GaAs lattice parameter $a$. 
comprises a $2 \times a$ thick $\operatorname{In}_{0.45} \mathrm{Ga}_{0.55} \mathrm{As}$ transition layer between WL and the QD apex. The third model QD depicted in Fig. 3(c) exhibits the same vertical chemical composition profile as that of Fig. 3(b), but has an inverted-cone shape. Previous investigations have shown that the in-plane symmetry of the QDs must be at least fourfold, ${ }^{12}$ so elongated objects were not considered. Our assumption of the In content increasing from $45 \%$ to $60 \%$ in the QD apex approximates a steadily increasing In proportion as previously proposed for nonburied InGaAs QDs, based on the evaluation of grazingincidence $\mathrm{X}$-ray diffraction measurements. ${ }^{14}$

The calculated intensity distributions of the three QDs [Figs. 3(a)-3(c)] are shown in Figs. 1(a)-1(c), respectively. All simulations reproduce the periodicity of the fivefold QD stack. However, Fig. 1(a) referring to a lens-like QD without transition layer, does not describe any detail of the diffuse scattering from the dots. This is particularly obvious if the measured features $P 1$ and $P 2$ are compared to the simulation. In contrast to Fig. 1(a), Figs. 1(b) and 1(c) referring to the lens-like and inverted-cone QD shape with transition layer, respectively, reflect the particular shapes of $P 1$ and $P 2$ and also other dot-related scattering intensity distributions very well. The rough approximation of a gradually increasing concentration profile by just a single step may be justified by the fact that the exact characteristics of the vertical profile essentially affects the intensities of high-order satellites only. Such high-order satellites are not shown here, because variations of their intensities cannot be resolved experimentally due to their low signal strengths. The remarkable similarity of Figs. 1(b) and 1(c) shows that the diffusely scattered $\mathrm{X}$-ray signal of the measured low-order satellites is largely insensitive to variations of the QD shape. Therefore, the QD shape could not be determined further using the available data. In spite of such experimental limitations, our findings clearly indicate a nonhomogeneous vertical In concentration which increases towards the QD apex. During formation, the upper parts of uncovered QDs can elastically relax well, since no lateral constraints prevent the lattice to expand. Therefore, the QD strain decreases from the bottom to the top of the QD. As long as the QDs are uncovered, lateral In migration from the WL to the QDs, as well as vertical In segregation from the QD bottom to the apex, ${ }^{15}$ are both favorable in terms of strain energy.
In summary, high-resolution diffuse x-ray scattering from five closely stacked InGaAs/GaAs quantum dot layers shows a complex intensity pattern which can be well described by dynamical scattering simulations if a vertical In concentration is assumed, which increases from the wetting layer towards the dot apex. A low In concentration in the WL as determined from the data indicates a migration of In from the WL to the QD apex during QD formation.

The authors gratefully appreciate the help of W. Drube (HASYLAB) during experiments. This work was supported by the Deutsche Forschungsgemeinschaft in the framework of Sonderforschungsbereich 296 projects A3 and A7, and by Project No. KO1510/2. R.L.S. acknowledges funding by Bookham Technologies.

${ }^{1}$ D. Bimberg, M. Grundmann, and N. N. Ledentsov, Quantum Dot Heterostructures (Wiley, Chichester, NY, 1999).

${ }^{2}$ V. A. Shchukin, N. N. Ledentsov, and D. Bimberg, Epitaxy of Nanostructures (Springer, New York, 2004).

${ }^{3}$ D. Bimberg and N. N. Ledentsov, J. Phys.: Condens. Matter 15, R1 (2003).

${ }^{4}$ P. W. Fry, I. E. Itskevich, D. J. Mowbray, M. S. Skolnick, J. J. Finley, J. A. Barker, E. P. OReilly, L. R. Wilson, I. A. Larkin, P. A. Maksym M. Hopkinson, M. Al-Khafaji, J. P. R. David, A. G. Cullis, G. Hill, and J. C. Clark, Phys. Rev. Lett. 84, 733 (2000).

${ }^{5}$ P. Jayavel, H. Tanaka, T. Kita, O. Wada, H. Ebe, M. Sugawara, J. Tatebayashi, Y. Arakawa, Y. Nakata, and T. Akiyama, Appl. Phys. Lett. 84, 1820 (2004).

${ }^{6}$ N. Liu, H. K. Lyeo, C. K. Shih, M. Oshima, T. Mano, and N. Koguchi, Appl. Phys. Lett. 80, 4345 (2002).

${ }^{7}$ N. Liu, J. Tersoff, O. Baklenov, J. A. L. Holmes, and C. K. Shih, Phys. Rev. Lett. 84, 334 (2000).

${ }^{8}$ A. Lenz, R. Timm, H. Eisele, C. Hennig, S. K. Becker, R. L. Sellin, U. W. Pohl, D. Bimberg, and M. Dähne, Appl. Phys. Lett. 81, 5150 (2002).

${ }^{9}$ D. Grigoriev, M. Hanke, M. Schmidbauer, P. Schäfer, O. Konovalov, and R. Köhler, J. Phys. D 36, A225 (2003).

${ }^{10}$ M. Hanke, M. Schmidbauer, D. Grigoriev, P. Schäfer, R. Köhler, A.-K. Gerlitzke, and H. Wawra, Phys. Rev. B 69, 075317 (2004).

${ }^{11}$ R. L. Sellin, F. Heinrichsdorff, C. Ribbat, M. Grundmann, U. W. Pohl, and D. Bimberg, J. Cryst. Growth 221, 581 (2000).

${ }^{12}$ M. Hanke, D. Grigoriev, M. Schmidbauer, P. Schäfer, R. Köhler, U. W. Pohl, R. L. Sellin, D. Bimberg, N. D. Zakharov, and P. Werner, Physica E (Amsterdam) 21, 684 (2004).

${ }^{13}$ A. Krost, F. Heinrichsdorff, D. Bimberg, A. Darhuber, and G. Bauer, Appl. Phys. Lett. 68, 785 (1996).

${ }^{14}$ I. Kegel, T. H. Metzger, A. Lorke, J. Peisl, J. Stangl, G. Bauer, K. Nordlund, W. V. Schoenfeld, and P. M. Petroff, Phys. Rev. B 63, 035318 (2001).

${ }^{15}$ L. G. Wang, P. Kratzer, N. Moll, and M. Scheffler, Phys. Rev. B 62, 1897 (2000). 